\title{
Deleuze et les Stoïciens: une logique de l'événement
}

\section{Sean Bowden}

Nous savons que, pour Deleuze, et bien qu'il soit ontologiquement antérieur aux « choses ", l'événement est avant tout un problème pour la pensée. Dès les premières pages de Logique $d u$ sens, Deleuze nous présente une série de "paradoxes événementiels " auxquels, comme une sorte de toile de fond, toutes les caractérisations positives de la nature des événements qu'il avance apparaittront comme des réponses dotées de sens. ${ }^{1}$ Qui plus est, nous savons que Deleuze fait appel à la philosophie des Stoïciens, parmi d'autres, afin de formuler cette ontologie - voire cette problématologie - des événements. Malheureusement pourtant, la nature de cet «appel » spécifique n'a pas encore reçu dans la littérature l'attention qu'il mérite. Par conséquent, cet essai prétend viser deux objectifs. Tout d'abord, il s'agira d'élaborer brièvement ce qui nous semble être les paradoxes événementiels les plus pertinents dans Logique du sens. En second lieu, il s'agira d'examiner et d'expliquer ce que Deleuze retire des Stoïciens en réponse à ces paradoxes. Ainsi, nous allons suivre la signification de la distinction ontologique que font les Stoïciens entre les corps et les événements et, par la suite, examiner comment cette distinction fonctionne dans les différentes parties de leur philosophie: physique, logique et éthique. Cette dernière étape sera nécessaire car Deleuze s'inspire précisément des Stoïciens lorsqu'il affirme que le statut particulier ontologique de l'événement implique une certaine éthique coextensive avec l'Être (la 


\section{DELEUZE ET LES STOÏCIENS}

Nature) et la pensée, c'est-à-dire coextensive avec un certain « usage vivant » des représentations logiques face au problème du devenir.

Pour commencer, reconstituons la problématique de Deleuze dans Logique du sens. Il faut dire tout d'abord qu'une notion d'événement est nécessaire pour parler d'un changement dans un état de choses, c'est-à-dire pour exprimer le passage d'un état déterminé à un autre. Étant donné que les choses changent et deviennent, il faut avoir une notion d'événement pour parler d'un avant et d'un après le changement, si petit soit-il. C'est parce que cet avant et cet après devraient toujours être tous les deux logiquement postérieurs à un événement ou à un devenir que l'on pourra dire qu'il a eu lieu. Un événement est un changement par lequel se constitue un nouveau présent, et par lequel un ancien présent se constitue comme passé. ${ }^{2} \mathrm{D}$ 'après la première série de Logique du sens, par exemple, on ne peut pas être plus grand qu'auparavant sans supposer un événement de "grandir", quelle que soit l'ampleur de ce changement. Le problème, toutefois, est que l'événement lui-même, comme antérieur aux états de choses, à l'avant et à l'après qu'il constitue, n'a pas d'identité assignable. En effet, il met les deux états l'un dans l'autre, ou plutôt il exprime la simultanéité de leur constitution, et dans la mesure où il est quelque chose, un devenir par exemple, il ne peut « être » en lui-même que l'identité infinie de ces deux états différents (LS, 9-12). Étant en lui-même contradictoire, il n'est donc pas. C'est ainsi que, selon l'exemple de Deleuze, l'événement "grandir ", exprimé dans la proposition « Alice grandit », implique à la fois un devenir plus grand qu'auparavant, et un devenir plus petit que maintenant (LS, 9). Ou considérons un événement comme "tuer lentement par empoisonnement ": il implique que le meurtre a lieu au même instant que la mort, ou encore que le meurtre est cause de la mort autant que la mort est cause du meurtre. Sans la mort, il n'y a pas de meurtre, bien que le meurtre soit cause de la mort. Encore une fois pourtant, on ne sortira pas de ce problème en distinguant les moments temporels différents, car ces moments, en tant que différents les uns des autres, doivent se constituer ou être différenciés par l'événement même. ${ }^{3}$ Pour autant donc que l'événement est " en cours ", il défait ou diffère toute détermination. C'est le paradoxe du pur devenir selon lequel les choses ne se succèdent plus dans un ordre universel linéaire, et à l'intérieur duquel il est impossible d'assigner des identités fixes (LS, 9-12). 


\section{SEAN BOWDEN}

Mais, en revanche, un événement n'est aussi qu'un effet, pour ainsi dire, de ces deux états de choses que l'événement à la fois détermine et met l'un dans l'autre, en ce sens qu'ils fixent, comme individualisés, les limites de l'événement: son commencement et sa fin par exemple (LS, 10-11). On ne pourrait parler d'un événement concret de " grandir» sans parler aussi d'un «avoir été plus petit » assignable et d'un «être maintenant plus grand qu'auparavant » mesurable. Afin de déterminer alors l'événement, est-il possible de le ramener à un état de choses original, une « image » de l'éternité ou d'un ordre universel, dont chaque événement et donc chaque état de choses serait logiquement l'effet ou l'expression partielle mais assignable? Non plus, semble-t-il, car toute tentative d'établir cette «image » nous ramène au paradoxe dont on voulait s'extraire, c'est-à-dire à l'événement de son établissement: soit objectivement, pour autant que tout commencement, toute fondation, doit impliquer un avant et un après; soit subjectivement, puisque dire ce qu'est enfin l'événement suppose aussi la détermination de ce qu'est l'événement dire. $^{4}$

Reste donc toujours l'événement paradoxal: ontologiquement nécessaire et pourtant contradictoire en lui-même; avancé à la fois comme "cause » et comme "effet» des états de choses; toujours supposé mais dont la détermination est infiniment différée. Qu'est-ce que cet événement? Comme Michel Foucault l'a écrit, afin de considérer cet événement, on doit d'abord lui donner une base métaphysique. ${ }^{5}$ Mais il faut avoir une ontologie structurée tout à fait différemment de celle qui dépend de l'affirmation d'un ordre fixe et éternel auquel le langage des événements peut correspondre, ou qui sert, dans son identité supposée, de médiateur pour ce langage. Et c'est des Stoïciens que Deleuze prend les premiers éléments de cette ontologie: premièrement, la distinction qu'ils font dans leur ontologie entre les corps et les événements « incorporels », tous les deux étant objectivement « quelque chose 1; et deuxièmement, un certain relais de rapports-physiques, logiques et éthiques-entre les corps et les événements qui prétend établir une systématicité complète et vivante, égale d'une certaine façon à l'événement du kosmos ou au tout lui-même.

Notre tâche principale ici est alors d'examiner ce que Deleuze retire des Stoïciens. Nous allons suivre un ordre systématique d'explication. Nous allons résumer le système stoïcien de manière à en rendre claire la lecture deleuzienne qui n'apparait que dans une forme fragmentée et discontinue dans Logique du sens. Dans cette élaboration, nous allons nous référer aux sources stoïciennes, à plusieurs 


\section{DELEUZE ET LES STOÏCIENS}

commentateurs y compris Victor Goldschmidt et Michael Frede, et enfin, pour les expliquer et les situer dans leur contexte, aux gloses souvent brèves de Deleuze. Munis de ce résumé, nous serons alors capable de montrer précisément de quelle manière les Stoïciens fournissent à Deleuze des réponses aux paradoxes de l'événement tels que nous les avons vus ci-dessus, bien que, finalement, Deleuze dépasse la solution stoïcienne. Cette dissertation constitue donc non seulement une étude d'histoire de la philosophie, mais encore fera partie de l'ensemble d'études sur Deleuze, dans la mesure où elle vise à expliquer ce que Deleuze emprunte aux Stoïciens et pourquoi, à la lumière du système stoïcien et aussi des préoccupations philosophiques de Deleuze. Notons pourtant que, dans une dissertation assez brève, nous ne pouvons apporter toutes les précisions qui seraient nécessaires à une étude détaillée des sources stö̈ciennes. Nous traitons donc le corpus stoïcien comme un ensemble, cohérent dans ses grandes lignes, tout en reconnaissant que cela constitue peut-être un abus.

\section{Ontologie stoïcienne}

Comme l'explique Deleuze, le terme le plus haut dans l'ontologie stoïcienne n'est pas l'Etre, mais Quelque chose, ti, qui subsume, d'une part, les corps individuels qui seuls sont les êtres réels et existants; et d'autre part, les incorporels qui subsistent, étant objectivement « quelque chose ", mais n'existent pas du tout (LS, 16). Les " universaux ", toutefois, n'ont pas de place parmi les "quelque choses »: ils ne sont que de purs "phantasmes ", et n'existent ni ne subsistent. ${ }^{7}$

Un corps, pour les Stoïciens, est ce qui peut agir ou pâtir, et tout corps a comme source deux principes éternels qui sont eux-mêmes des corps: la matière passive et le Dieu actif dans cette matière ( $~$ feu artiste " ou logos). Ensemble, ils forment un tout, le kosmos ou le monde. ${ }^{8}$ Peuplent ce monde des corps de toutes sortes. L'âme est un corps, par exemple, car elle peut occasionner la souffrance du corps avec lequel elle interagit, lorsqu'elle éprouve de la crainte par exemple. ${ }^{9}$ Les qualités comme la sagesse ou les vertus sont des corps, ou des états complexes physiques, car leur possession suscite un certain effet. La possession

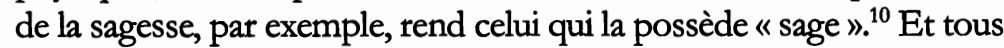
les corps sont unifiés et se développent, les uns mêlés totalement avec les autres tout en conservant leurs substances propres, par un " souffle » divin ou un mouvement tensionnel qui pénètre et traverse l'univers tout entier, et qui détermine, d'une conflagration à une autre, tout corps, 


\section{SEAN BOWDEN}

leurs manières d'être et les états de choses correspondants. ${ }^{11}$ Ainsi, le souffle divin ou l'activité de Dieu est la cause sustentatrice de tout individu dans une sympathie universelle, le destin ou le présent existant cosmique, où les corps, suivant leurs propres natures ou tensions, sont des causes les uns pour les autres de tout ce qui se passe $(\mathrm{LS}, 13){ }^{12}$

Les incorporels, en revanche, n'agissent ni ne pâtissent. À l'opposé des Idées de Platon, les incorporels stoïciens n'ont pas d'efficacité causale. Ils ne sont pas des êtres physiques, mais ils subsistent objectivement. Ils sont quatre: le dicible ou l'exprimable (lekton), le vide, le lieu et le temps. ${ }^{13}$ Mais pourquoi cette division ontologique entre les corps et les incorporels? Nous montrerons que, point capital pour Deleuze, cette division dans l'ontologie stoïcienne répond au problème des événements, c'est-à-dire au problème de penser d'une façon cohérente les choses et leurs devenirs, sans recours à quelque chose échappant à l'enchaînement causal rigoureux qui seul pourrait être tenu pour responsable de tel ou tel devenir dans le monde. Avant d'examiner comment fonctionne cette division ontologique dans les différentes parties du système stoïcien, examinons brièvement comment chacun des incorporels répond ainsi à un problème événementiel.

Prenons d'abord le vide et le lieu. Le vide comme incorporel, suivant Victor Goldschmidt, répond au problème de concevoir l'événement du kosmos lui-même avant chaque conflagration, dans la mesure où concevoir le monde implique aussi « concevoir ce lieu, avant que le monde ne l'appelle à l'existence..., destitué de toute qualité. Et dont on peut dire "qu'il est simplement capable de recevoir un corps", ou "d'être occupé par un corps" $\gg .{ }^{14} \mathrm{Le}$ lieu se définit donc par contraste comme ce qu'un corps peut occuper et occupe effectivement, ou ce qui peut être partiellement rempli et partiellement vide par rapport à un corps particulier, sans pour autant être lui-même un corps. ${ }^{15}$ Le lieu est donc comme « un thêâtre toujours rempli où des corps se succèdent ou se compénètrent $"{ }^{16}$ Le vide et le lieu, en tant qu'incorporels objectifs subsistants, répondent alors ainsi au problème de la pensée non exhaustive de tous les changements et les rapports spatiaux entre tout ce qui existe: les corps.

Prenons maintenant le temps. Pour Chrysippe, il s'agit de "l'intervalle du mouvement» du monde. Autrement dit, Chrysippe comprenait le temps comme dépendant de l'existence du mouvement lui-même qui, en dernière analyse, est coextensive avec le mouvement du monde physique. Et pourtant, nous trouvons une contradiction apparente dans la conception chrysippéenne du temps. Il dit, d'une 


\section{DELEUZE ET LES STOÏCIENS}

part, qu'« aucun temps n'est complètement présent »; mais d'autre part que « seul le présent est le cas [buparchein]; le passé et le futur subsistent, mais ne sont nullement le cas $"{ }^{17}$ Goldschmidt interprète cette conception du temps, et cette contradiction apparente, où, d'un côté, aucun temps n'est présent en toute rigueur de termes et, de l'autre, il y a effectivement une différence entre le présent « existant » et « le passé et le futur subsistants ", de la manière suivante: il ne faut pas confondre ce qui seul existe, le mouvement du monde, avec la pensée de ce qui arrive qui n'est qu'une expression partielle du présent cosmique. ${ }^{18}$ Autrement dit, pour les Stoïciens, il n'y a que le mouvement cosmique qui soit réel, le destin qui ne demeure présent qu'à Zeus. Tout événement, tout ce qui arrive est, réellement, le résultat d'un entrelacement des corps et des causes dans la sympathie universelle. Mais pour nous, les êtres limités dans le monde, ne voyant pas comme Zeus, ce qui arrive n'est pensable qu'à partir d'un instant temporel idéal qui se divise en un passé et un futur subsistants et incorporels. A quel problème événementiel répond alors ainsi l'incorporalité du temps? En bref, sans la différence entre le présent existant des corps et le temps incorporel, les Stoïciens n'auraient pas pu dire que, de notre point de vue, chaque événement temporel se ramène au même ordre, soit à la juste mesure du présent cosmique. Suivant l'analyse indirecte de Deleuze, s'ils avaient admis la corporalité du temps ou de l'événement temporel, ils auraient dû aussi admettre que chaque mouvement relatif dans le passé ou le futur, pris au hasard, serait capable de modifier le tout (voir LS, 190-194). Dans les termes de nos exemples, « en un seul et même abîme qui menace le présent et tout ce qui existe ", voire dans un présent devenu "démesuré » (LS, 192), autant que l'inverse, « mourir » devrait déterminer « tuer » et « rapetisser » devrait précéder "grandir ». L'incorporalité du temps rend alors pensable, à l'intérieur d'un seul présent vivant, les rapports relatifs entre des états de choses temporels diversement conçus.

Examinons enfin « l'exprimable » ou « le dicible » stoïcien auquel Deleuze s'intéresse surtout, et qui nous concerne principalement ici. Pour les Stoïciens, lorsqu'un corps agit sur un autre corps, il produit un effet, un événement spatial et temporel qui n'est pas un autre corps à proprement parler, mais un prédicat incorporel, un exprimable, un verbe, un attribut logique ou dialectique qui subsiste dans la pensée (LS, 13). "Par exemple, le scalpel est un corps, qui devient pour la chair, autre corps, cause du prédicat incorporel "être coupé" $\gg{ }^{19}$ Mais pourquoi cette distinction ontologique entre un corps existant et ses 


\section{SEAN BOWDEN}

prédicats incorporels? Comme le suggèrent Long et Sedley, cette distinction résout le problème de la conceptualisation du rapport entre un corps individuel existant et ses changements. C'est parce que, si la chair intacte cessait d'exister après l'événement de couper, remplacée ainsi par un nouveau corps, la chair coupée, cela impliquerait alors qu'aucun corps ne persiste au long du processus. Puisque l'effet ne saurait donc être un nouveau corps, il doit être un prédicat incorporel qui vient à être vrai de la chair, laquelle persiste. ${ }^{20}$

Deleuze, pour sa part, suggère que l'exprimable incorporel répond au problème de ce qui se dérobait à l'action de l'Idée chez Platon: le devenir ou les simulacres comme " grandir ». Au lieu de repousser alors les devenirs qui esquivent et menacent le présent dans la profondeur des corps et de faire de l'Idée la seule réalité, l'événement est maintenant incorporel, dépourvu de toute activité réelle et pourtant représentant toute idéalité (LS, 17). Et nous verrons plus tard le rôle que joue l'idéalité de l'exprimable dans le système stoïcien qui est surtout un ars vitae face à ce qui arrive. Mais nous pouvons dire ici que les incorporels en général correspondent, pour Deleuze, aux aspects de l'événement paradoxal que l'on a examinés ci-dessus: à la fois déterminant pour la pensée et effet des états de choses; inassignable en tant que " quelque chose » et pourtant objectivement nécessaire, subsistant en tant que tel dans les propositions qui l'expriment. Comme Goldschmidt l'a dit, pour les Stoïciens, c'est toujours le corps, et enfin le corps unique ou le présent cosmique, qui fait que les incorporels " prennent corps $» .{ }^{21}$ Mais cela revient aussi à dire que seuls les corps sauraient déterminer que les incorporels rendent les corps et leurs devenirs déterminables pour la pensée. C'est dire que la détermination de l'événement appartient aux corps, mais aussi que tout état de corps est lui-même déterminé pour la pensée par l'événement « exprimable » dans la proposition. Or, ontologiquement, l'événement ne se laisse jamais réduire à un corps, ou inversement. Donc, bien que nous ayons une première réponse aux paradoxes événementiels tels que nous les avons examinés ci-dessus, cet écart irréductible ontologique entre les corps et les événements exige évidemment aussi une systématisation du maniement de l'evénement, c'est-à-dire une systématisation qui lie ensemble, pour autant qu'il s'agit de le penser et de le vivre, une physique, une logique et une éthique de l'événement (éthique dans le sens des règles de conduite que l'événement nécessite). Et c'est pourquoi il faut maintenant examiner comment la distinction ontologique entre les corps et les incorporels fonctionne dans les différentes divisions de la philosophie stoïcienne, et aussi, plus 


\section{DELEUZE ET LES STOÏCIENS}

important, ce que Deleuze en retire. Notons ici toutefois que pour Deleuze, même lorsqu'il use de concepts stoïciens, il s'agira de penser et de vivre l'événement au-delà de toute unité cosmique ou " présence » divine.

\section{Le système stoïcien}

Les Stoïciens divisent la philosophie en trois parties qui sont coordonnées systématiquement les unes aux autres: physique, logique et éthique.22 Effectivement, chaque partie de leur philosophie se fonde sur des thèses empruntées aux autres parties. D'une part, par exemple, la fin éthique, "vivre en accord avec la nature ", requiert une compréhension du rôle causal de Dieu aussi bien qu'une compréhension des règles du raisonnement sur lui. D'autre part, si l'on avait demandé aux Stoïciens à quelle fin aboutissent leurs recherches en physique et en logique, ils auraient répondu que la dialectique et la physique ne sont pas à proprement parler des moyens vers quelque chose: elles sont plutôt des vertus dont l'unité est identique à la sagesse ou à l'âme raisonnable, ou encore vertueuse, du sage. ${ }^{23}$ Comment fonctionnent alors plus précisément ces divisions de la philosophie, surtout en ce qui concerne la distinction ontologique entre les corps et les incorporels, et dont la place de l'exprimable est avant tout la préoccupation primaire de Deleuze? Pour aborder les éléments de réponse, nous commencerons par la physique.

\section{La physique}

La physique pour les Stoïciens se pratique chaque fois qu'ils font des recherches sur le monde et sur ce qu'il contient. ${ }^{24}$ Comme nous l'avons vu en examinant ci-dessus ce qu'est un corps chez eux, la physique stoïcienne, qui approche de ce que nous appelons aujourd'hui une métaphysique de la nature, ${ }^{25}$ affirme l'existence d'un monde unique et unifié, un kosmos vivant qui se compose entièrement des corps qui agissent et pâtissent, et qui sont des causes les uns pour les autres de tout ce qui arrive. Les deux principes (Zeus et la matière), les quatre éléments, les plantes et les animaux, les âmes et les qualités comme la dureté et la sagesse, sont tous des corps qui dans leur hiérarchie, leur interpénétration, leurs dispositions, actions et passions, sont les raisons réelles de ce qui se passe. Effectivement, la physique stoïcienne fournit ces conceptions et ces distinctions métaphysiques afin d'expliquer comment l'univers fonctionne selon un plan divin et rationnel. Le 


\section{SEAN BOWDEN}

physicien « rend compte de l'univers à l'aide de concepts métaphysiques qui, remontant à l'origine première des choses, c'est-à-dire en dernière instance à l'Agent, ont le pouvoir de tout expliquer $»{ }^{26} \mathrm{Ce}$ qui intéresse pourtant Deleuze, plutôt que la théologie rationnelle des Stoïciens, c'est la division dans la physique stoïcienne entre les causes et les effets, ou encore entre les corps et les incorporels, division qui, selon lui, présente une solution au problème du devenir (LS, 13-16).

Les Stoïciens placent alors toute réalité dans les corps vivants et distinguent ces réalités des manières d'être qu'elles nous présentent, qui ne sont que des abstractions ou des signes, c'est-à-dire, à la fois, des effets incorporels des actions et des passions des corps et, dans le cas de l'exprimable, le matériau de la logique stoïcienne. Il s'agit donc de chercher la raison immanente des choses dans les choses elles-mêmes, à l'aide de leurs effets incorporels, des signes, qui n'ont pas d'efficacité causale, mais seulement une certaine subsistance pour la pensée (LS, 13-18). ${ }^{27}$ Les incorporels qui sont le temps, le lieu et le vide, comme nous l'avons vu, dépendent entièrement des corps pour leur statut objectif. Dans la mesure où ils se ramènent au mouvement du monde vivant qui les « occupe » (ou qui peut les occuper dans le cas du vide), ils ne sont proprement que les effets de ce mouvement, bien que l'on ait besoin de ces incorporels, comme des "corrélats " des corps, pour penser le mouvement dans la mathématique physique. ${ }^{28}$ Quant à l'incorporel qui est l'exprimable, il relève, d'une part, d'une analyse causale des corps et, d'autre part, comme nous allons le voir, de la dialectique stoïcienne. L'exprimable n'est donc pas à strictement parler un corrélat des corps, puisqu'il suit d'autres lois que celles des corps. Et pourtant, l'exprimable doit aussi se produire entièrement par les corps, en ce que toute réalité est corps. Voyons comment s'effectue cette analyse causale et logique de l'exprimable.

Les Stoïciens distinguent, non seulement entre la cause et l'effet, mais encore entre plusieurs espèces de causes: causes sustentatrices, complètes, prochaines, auxiliaires, coopérantes, antécédentes, et cæetera. ${ }^{29}$ Or, comme Michael Frede l'a montré, parmi toutes ces causes, les Stoïciens veulent toujours distinguer ce qui est la cause à proprement parler, c'est-à-dire, la cause complète (autoteles) ou sustentatrice (sunektikon). ${ }^{30} \mathrm{Et}$ cette cause complète ou sustentatrice doit toujours être un corps: puisqu'ils n'ont pas possédé la notion de l'inertie de la matière, les Stoïciens mettaient toute force et toute activité à l'intérieur des corps individuels. ${ }^{31}$ Cela veut dire alors que, pour chaque événement, ils veulent identifier « ce qui est responsable » (aition), quel corps par sa 


\section{DELEUZE ET LES STOÏCIENS}

propre action a produit l'événement. ${ }^{32}$

Or, l'événement, ce qui est produit, n'est pas un autre corps, mais un prédicat, un exprimable qui vient à être vrai du corps. ${ }^{33}$ C'est donc du fait de la nature du bois, par exemple, que «le bois brûle » ou que «le bois se réduit en cendres ». Le bois est cause complète des prédicats qui viennent à en être vrais. Toutefois, ce n'est pas encore suffisant, car la représentation du rapport causal n'est pas entre un corps et un prédicat, mais entre un corps, un deuxième corps, et un prédicat qui est vrai de ce corps-ci. Le feu, un corps, devient pour le bois, autre corps, cause du prédicat incorporel "être brûlé ", par exemple. ${ }^{34}$ Comment donc analyser ce rapport, étant donné que les Stoïciens doivent apparemment affirmer l'existence des causes externes, par l'opposition à leur thèse que toute force et toute activité sont internes au corps?

En fait, les Stoïciens affirment l'existence des causes antécédentes. Pour eux, rien n'est séparé de tout ce qui s'est produit auparavant, car sinon, le monde serait divisé, écartelé, et ne resterait plus une unité. ${ }^{35}$ Encore une fois alors, comment réconcilier la notion des causes antécédentes avec la thèse que toute force est interne au corps? Comme Frede l'a montré, le rapport causal doit être analysé en au moins deux rapports, chacun entre une cause complète et son effet. Ainsi, toute cause antécédente est cause antécédente de quelque chose, $p$, en étant cause complète [sunektikon] pour une cause complète, $s$, d'une affection passive, $q$, d'une façon telle qu'une cause complète s en état $q$ soit cause complète de $p .36 \mathrm{Par}$ exemple, une fois poussé, un cylindre roule par sa propre disposition, mais ce n'est que moi qui peux pousser, et ce n'est que le cylindre qui peut rouler. Le cylindre n'est donc cause complète active du prédicat « rouler » qu'en étant passif par rapport à moi. Je suis donc la cause complète pour que le cylindre soit cause complète, du fait de sa propre nature ou affection, de l'exprimable « rouler ».37

Et c'est précisément ainsi que Deleuze analyse la physique stoïcienne: la passion d'un corps, dit-il, renvoie toujours à l'action d'un corps plus puissant jusqu'au point du présent cosmique, Zeus, qui est cause active sustentatrice, en dernière instance, de tout ce qui se passe (LS, 190-191). Mais ce n'est pas que Zeus soit cause directe de chaque événement; ce n'est plutôt qu'à partir de cette unité de Zeus, du présent cosmique, que les corps se trouvent, par leurs propres natures dans le tout, des causes les uns pour les autres de tout ce qui se passe. C'est en ce sens, comme le dit Deleuze, que le présent cosmique mesure les actions 


\section{SEAN BOWDEN}

et les passions des corps. La mesure des parties dépend de la mesure du tout, même si le tout doit immanquablement nous échapper ou être cherché ailleurs, dans un autre élément comme nous allons le voir, logique et enfin "éthique » (LS, 190-191). Notons en passant aussi que, étant donnée une telle analyse causale, les Stoïciens peuvent affirmer le destin, tout en conservant la responsabilité morale, car c'est nous qui sommes responsables, de telle ou telle manière, de nos actions. ${ }^{38} \mathrm{Le}$ rapport avec la cause physique ainsi expliqué, voyons maintenant quel rapport a l'exprimable avec la logique stoïcienne.

\section{La logique}

En effet, le fondement de la logique stoïcienne-étant logikê, le savoir des fonctions de logos ou de raison-est lui-même une théorie des exprimables ou lekta. Comme nous l'avons vu, les exprimables sont des événements, des prédicats ou des verbes qu'un corps cause pour un autre corps, comme "être brûlé » ou "être coupé ». Mais en même temps, l'exprimable n'est pas un corps, il est quelque chose qui peut être dit au sujet d'un corps. ${ }^{39}$ Les lekta sont en effet des états de choses qui sont dits, et subsistent en conformité avec une représentation rationnelle (nous allons voir ce qu'est une représentation rationnelle). ${ }^{40}$ Dans la mesure où il se dit au sujet d'un corps, l'exprimable se distingue non seulement du "porteur du nom» ou le référent, mais encore nécessairement de l'émission vocale qui est aussi corps. ${ }^{41}$ Évidemment, on peut prononcer un discours sur une émission vocale de diverses manières, ce qui veut dire que l'exprimable est quelque chose comme le sens de ce qui se dit, sans être pour autant localisable dans ce qui se dit.

Pour être plus précis, les exprimables, en tant que dits, se divisent en exprimables incomplets ou inachevés, qui sont des prédicats, ou des verbes sans sujet qui peuvent être assertés; et en exprimables complets ou achevés où le prédicat est combiné avec un sujet, le cas nominatif que "porte" le référent, pour la genèse des propositions simples ou complexes. ${ }^{42}$ Et enfin, ce n'est que l'exprimable complet, ou la proposition, qui peut être vrai ou faux par rapport au monde. ${ }^{43}$ La question pour la logique est alors celle de savoir combiner les exprimables ou les événements pour générer des propositions vraies, ou pour savoir quand un prédicat peut en vérité être attribué à son sujet. Ou encore, il s'agit de penser le corps qui « porte » le cas nominatif, ainsi que les rapports avec d'autres corps, passifs ou actifs eu égard à ce premier, à partir de la combinaison des événements qui leur arrivent 


\section{DELEUZE ET LES STOÏCIENS}

tous. Comme nous allons le voir, les Stoïciens répondent à cette question en spécifiant les conditions sous lesquelles un événement peut être dit logiquement compatible ou incompatible avec un autre; et aussi en élaborant une théorie de la connaissance qui implique, éthiquement, de même que logiquement et physiquement, un certain usage des « représentations». Nous abordons ici premièrement les conditions logiques de compatibilité entre événements.

Comme nous venons de le voir, pour les Stoïciens, une proposition qui est un exprimable complet énonce un événement: « il fait jour » ou «Dion marche » par exemple. Or, ces propositions peuvent avoir des relations de différentes natures les unes avec les autres, constituant ainsi des propositions complexes. Une proposition conditionnelle, par exemple, a la forme "S'il fait jour, il fait clair »; la proposition conjonctive est de la forme «Et il fait jour, et il fait clair »; et l'exemple que l'on nous donne de la proposition disjonctive est « $\mathrm{Ou}$ bien il fait jour, ou il fait nuit ${ }^{44}{ }^{44} \mathrm{La}$ proposition conjonctive est vérifonctionnelle en ce qu'elle est vraie si tous les éléments qu'elle conjoint sont vrais par rapport au monde. ${ }^{45}$ La proposition disjonctive, toujours interprétée par les Stoïciens comme une disjonction exclusive, est vraie si l'un et seulement l'un des éléments disjoints est vrai en correspondant au monde. ${ }^{46} \mathrm{Ce}$ qui détermine qu'une proposition conditionnelle est vraie, pourtant, c'est que «l'opposé du conséquent est en conflit avec l'antécédent, par exemple: "S'il fait jour, il fait clair." Cela est vrai, car l'opposé du conséquent "Non: il fait clair", est en conflit avec "Il fait jour" $"{ }^{47}$ Mais la question est de savoir ce que signifie exactement la notion de "conflit » ou d'« incompatibilité »: est-elle à interpréter dans le sens d'une nécessité logique, ou d'une nécessité empirique ${ }^{48}$ Une réponse à cette question de "conflit » est d'autant plus importante en ce que la proposition conditionnelle nous fournit le modèle même pour atteindre à une vision du kosmos à partir des événements singuliers. ${ }^{49}$

Ce qui nous intéresse ici, c'est que Deleuze suggère que cette notion d'incompatibilité dérive de l'implication conceptuelle, ${ }^{50}$ mais aussi que celle-ci résulte toujours d'un processus d'une autre natureni conceptuel ni empirique à proprement parler-que nous devrons examiner ci-dessous (LS, 85-87; 199). En effet, il analyse le conditionnel stoïcien à partir d'une théorie des «types » où tout «nom » ou proposition reçoit une " détermination de signification " grâce à deux lois. Selon une première loi "régressive", chaque proposition est désignée par une autre proposition d'un « type » supérieur. Selon une 


\section{SEAN BOWDEN}

deuxième loi "disjonctive ", chaque proposition détermine des disjonctions remplies par d'autres propositions. Étant donné que « s'il fait jour, il fait clair ", il n'est donc pas possible qu'il fasse jour et qu'il ne fasse pas clair. C'est parce que « faire jour » devrait être élément d'un ensemble qu'il supposerait, et appartenir à l'un des groupes classés par rapport à lui (LS, 85-87; 199). En effet, c'est selon ces déterminations de signification, dérivées des deux lois, que l'on engendre les principes de non-contradiction de tiers exclu, au lieu de se les donner tout faits. Étant donné la hiérarchie des déterminations, il n'est pas possible qu'il fasse clair et qu'il ne fasse pas clair: ou bien il fait clair, ou bien il ne fait pas clair.

Et pourtant, selon Deleuze, pour soumettre ces noms ou ces propositions aux lois régressives et disjonctives, il faut avoir un nom ou une proposition auxquels les lois ne s'appliquent pas. C'est-à-dire, dans la mesure où chaque proposition est soumise à une détermination par une proposition de type supérieur, et où chaque proposition détermine des disjonctions remplies par d'autres propositions, il faut avoir une proposition qui se désigne elle-même ainsi que les propositions inférieures à elle, et qui détermine une disjonction dans laquelle elle entre. En bref, elle doit exprimer son propre sens, ce qui est proprement le non-sens. ${ }^{51}$ Comme le dit Deleuze, d'après les paradoxes de Russell, les deux figures du non-sens dans la signification conceptuelle sont: " qu'il y a un ensemble de tous les ensembles » et « qu'il y a le barbier du régiment 》 (LS, 84-86). Ce problème, comme nous l'avons suggéré, est celui de Logique du sens tout entier. C'est qu'en déterminant les rapports entre événements, la proposition « déterminante » en dernière instance prétend exprimer un événement alors qu'elle ne le peut pas: l'événement du tout dans lequel elle est déterminée aussi. Pour Deleuze, la philosophie stoïcienne représente une grande tentative pour éviter un tel paradoxe, ce qui veut dire que les Stoïciens, au moins tels que Deleuze voudrait les lire et comme nous allons le voir, nient un ordre conceptuel finalement déterminable d'implications entre exprimables ou événements.

L'intérêt de Deleuze pour la logique des Stoïciens est non seulement dans les connexions qu'ils font entre les propositions qui expriment les événements, mais aussi dans le rapport de ces connexions avec leur théorie de la modalité, ou plutôt la thèse stoïcienne qui affirme le destin physique mais qui nie la nécessité (voir LS, 18-19; 47; 86-87; 198-200). En effet, ces deux aspects de la logique des Stoïciens sont étroitement liés, car leur théorie de la modalité affirme la possibilité de 


\section{DELEUZE ET LES STOÏCIENS}

futurs contrefactuels précisément en ce sens que leurs propositions conditionnelles n'expriment ni un rapport réel causal ni un rapport conceptuel capable d'être prescrit. C'est pourquoi, comme l'explique Deleuze, les Stoïciens transforment les propositions des astrologues chaldéens qui prétendent annoncer la nécessité d'un événement futur à cause d'un événement déjà passé (et donc nécessaire, d'après Chrysippe) de leur forme conditionnelle en conjonction niée. La proposition chaldéenne «Si quelqu'un est né au lever de la Canicule, il ne mourra pas en mer » est donc ainsi transformée par les Stoïciens en «Non à la fois: quelqu'un est né au lever de la Canicule, et il mourra en mer ", par exemple. ${ }^{52}$ Selon Deleuze, cette transformation précise que, non seulement il n'y a pas de rapport réel de causalité physique entre les deux événements dont le conditionnel chaldéen voudrait exprimer le passage; mais encore qu'il n'y a pas d'implication conceptuelle nécessaire entre les deux événements. C'est parce que, selon ce qui précède, seules les propositions conditionnelles sauraient exprimer des implications conceptuelles entre événements, tandis que les propositions conjonctives, bien que transformées ici du conditionnel, sont vérifonctionnelles par rapport au monde, et leurs propositions constitutives peuvent changer leur valeur de vérité selon ce qui arrive effectivement. ${ }^{53}$ Qui plus est, en tout cas, le rapport causal ne serait jamais exprimé par le conditionnel, car seuls les corps sont causes et le conditionnel n'a comme objet que les combinaisons possibles de leurs effets (leurs «signes »). Et enfin la vérité de l'implication logique ne serait jamais exprimée par la vérité du conjonctif correspondant, car même la conjonction de "toute" proposition vraie manquerait, logiquement, une proposition "événementielle » qui déterminerait la conjonction totale en tant que telle. C'est finalement ce que veut dire la phrase énigmatique de Deleuze selon laquelle l'astrologie (sans doute des Chaldéens tempérée par des considérations stoïciennes) fut peutêtre la première grande tentative pour établir une théorie des incompatibilités alogiques et des correspondances non causales, en ce qu'il s'agit de la production et de la multiplication des liens événementiels ou "surfaces » idéales physiques, et de leur interprétation et leur orientation les unes vers les autres, au-delà de toute connexion nécessaire, afin de suivre le mouvement du destin ou de ce qui se passe effectivement (LS, 200; 168).

Nous pouvons maintenant voir clairement comment l'interprétation logique des événements est pour les Stoïciens ce corrélat d'une théorie de la divination physique, ou de l'interprétation des signes 


\section{SEAN BOWDEN}

«physiques » dont la forme, selon Sextus Empiricus, est précisément celle du conditionnel « si ceci, alors cela $\|^{54}$ Le rapport entre les signes est celui de l'implication. La transformation du conditionnel en proposition conjonctive, pourtant, nous renvoie précisément au présent cosmique, car les signes ou les événements sont signes de choses: les événements sont ce que les choses subissent ou provoquent les uns pour les autres dans un ordre cosmique qui est total et présent. Comme Deleuze note justement à cet égard, il arrive aux Stoïciens de dire que les signes sont toujours présents, et signes de choses présentes. Par exemple, de "celui qui est mortellement blessé, on ne peut pas dire qu'il a été blessé et qu'il mourra, mais qu'il est ayant été blessé, et qu'il est devant mourir » (LS, 79-80)..$^{55}$ Autrement dit, il s'agit toujours de traduire un lien de conséquence proposé en coexistence conjonctive, mais où une seule fausse proposition conjointe risque de gâcher le tout. ${ }^{56} \mathrm{Il}$ s'agit donc, à partir des événements singuliers, et les liens proposés entre eux, d'atteindre peu à peu à une vision de l'événement du kosmos, du présent unique.

Ce qui importe pour Deleuze est que, compte tenu des exigences de l'événement paradoxal examinées ci-dessus, il devient possible, selon les Stoïciens, de définir un va-et-vient rigoureux entre deux rapports dans la proposition qui exprime l'événement: le rapport entre la proposition et les états de choses correspondants, et le rapport d'implication entre les propositions diverses. D'une part alors, ce qui est posé par le rapport d'implication logique, exprimé par le conditionnel, présente autant de cas pour la combinaison d'événements. D'autre part, ce qui est indiqué par le rapport dénotatif, exprimé par la proposition conjonctive, joue le rôle d'instances potentielles matérielles, selon ce qui se passe, pour lesdites combinaisons événementielles (LS, 143-144). Autrement dit, nous semble-t-il, ce sera précisément ce vaet-vient que Deleuze, inspiré par les Stoïciens, affirmera comme élément de la production du "sens» en réponse au problème de déterminer l'événement, une production qui est au-delà de tout principe de contradiction et neutre à l'égard de toute modalité: « fatale » mais non nécessaire (LS, 47).

Or, en revenant au fil de notre exposé, que fonde et guide épistémologiquement ce va-et-vient? Quel rapport exact ont les événements exprimables avec les corps et le monde, étant donné qu'il ne s'agit pas strictement d'une causalité physique enfin devinable, ni d'une implication conceptuelle finalement déterminable? S'il s'agit de connaitre, dans telle ou telle situation, les autres événements avec lesquels 


\section{DELEUZE ET LES STOÏCIENS}

un événement particulier est « confatal » selon le destin, d'après quels critères ces événements sont-ils véritablement pour la connaissance des confatalia ou inconfatalia? ${ }^{57}$ Afin de voir comment les Stoïciens résolvent ce problème, il faut examiner, en premier lieu, leur épistémologie et leur concept d'une « représentation compréhensive $»{ }^{58}$

\section{Logique et épistémologie}

La théorie de la connaissance chez les Stoïciens, à proprement parler, fait partie de leur logique, car la logique est l'étude de tout ce qui a quelque chose à voir avec le discours rationnel, et pour les Stoïciens, " toutes choses se perçoivent par le biais d'une théorie raisonnée ", ce qui revient aussi à dire que la pensée et le jugement sont des modes du discours rationnel..$^{59}$ En effet, la théorie de la connaissance propose les critères de la vérité pour le jugement, ou encore, pour l'assentiment à une impression ou une représentation (phantasia) dans l'âme..$^{60}$

Résumons les aspects essentiels de la théorie stoïcienne. Tout d'abord, une représentation (phantasia) sensible est une affection ou une modification qui, à travers les organes sensoriels, se produit dans l'âme corporelle, et qui se révèle elle-même en même temps que ce qui l'a produite: "l'impresseur $»{ }^{61}$ Une telle représentation sensible est différente de la proposition qui la caractérise, puisqu'une proposition peut être la même pour des représentations sensibles diverses: impressions reçues de près ou de loin, et cætera. ${ }^{62}$ Une représentation rationnelle, par contraste, se forme dans l'âme par la pensée (noèsis), et, précisément, elle a un contenu qui peut être exhibé dans le langage sous la forme " $p$ est $q$ ", ce qui correspond à la forme d'un exprimable " complet ${ }^{63}$ C'est parce que, selon une conception providentialiste stoïcienne de la nature humaine, ce qui nous distingue des animaux non-rationnels c'est que nous pensons par transition (metabatike) et par combinaison (sunthetikè), c'est-à-dire correspondant aussi à la manière dont les exprimables ou les signes sont pensés. ${ }^{64}$

En tant que formée par la faculté directrice de l'âme ou l'esprit, la représentation rationnelle est nécessairement sensible aux caractéristiques particulières de l'esprit dont il s'agit: le maillage de ses autres représentations ou croyances par exemple. ${ }^{65}$ En ce sens alors, d'une part, les représentations rationnelles seront toujours corporelles, des états complexes physiques, puisqu'elles dépendent de l'expérience, c'est-à-dire l'accumulation de représentations sensibles. ${ }^{66} \mathrm{D}$ 'autre part, les représentations rationnelles appartiendront aussi toujours à la pensée dont la logique étudie les règles, en ce que le contenu de la représentation 


\section{SEAN BOWDEN}

est propositionnel et formé par transition et par combinaison. Comme l'a dit Frédérique Ildefonce, selon Claude Imbert, « la représentation contient de manière enveloppée toutes les informations que la proposition développera dans le champ de la discursivité effective $1 .^{67}$ Reste pourtant la question du critère de la vérité pour la connaissance; ou ce qui revient au même, la question de savoir à quel point la représentation corporelle et le jugement logique coïncident l'un avec l'autre.

Nous en arrivons alors au problème de l'impression cognitive ou compréhensive qui est précisément, pour les Stoïciens, le critère de la vérité. ${ }^{68}$ Une impression cognitive a trois conditions: elle " est une impression qui provient de ce qui est, et qui est scellée et imprimée en conformité exacte avec ce qui est, telle qu'elle ne saurait provenir de ce qui n'est pas $»{ }^{69} \mathrm{La}$ question est de savoir ce que signifie exactement " ce qui est ». Selon Michael Frede, il parait nécessaire de l'interpréter de la manière suivante: il faut comprendre « ce qui est », non pas comme l'objet réel, mais plutôt comme l'état ou la manière d'être de cet objet, le fait que cet objet «est tel ou tel», bien que ce fait ne puisse rien causer. Pour que la représentation $\mathrm{P}$ est $\mathrm{Q}$ soit compréhensive, elle doit avoir son origine dans le fait que $\mathrm{P}$ est $\mathrm{Q} .^{70}$ Pourtant, à quoi bon dire que la représentation compréhensive s'imprime dans l'esprit en conformité exacte avec "ce qui est ", si "ce qui est ", dépourvu de toute efficacité causale, est précisément ce que l'on cherche à connaittre à l'aide de la représentation compréhensive? ${ }^{71}$ Or, comme Frede l'a bien montré, la thèse stoïcienne de "l'identité des indiscernables » implique que la représentation compréhensive puisse en principe distinguer un objet de tout autre, et donc tout fait ou événement qui proprement lui appartient. ${ }^{72}$ Mais dans ce cas, la marque d'une représentation compréhensive ne sera rien au-delà de son énonciation de telle ou telle manière, et donc en rapport avec un maillage d'autres énonciations et leurs représentations corporelles correspondantes. ${ }^{73}$ Pour les Stoïciens alors, il s'agit de retranscrire avec une précision artiste, à partir des représentations ou des faits donnés, toutes les caractéristiques propres de la situation, c'est-à-dire, tous les corps du point de vue des événements ou faits dont ils sont causes les uns pour les autres jusqu'au point du présent cosmique qui est cause unique, et qui est identique à l'enchaînement entier des événements.

Voilà enfin ce qui intéresse principalement Deleuze, et qui nous renvoie une fois de plus à la problématique de Logique du sens, car la représentation doit envelopper à ce point une expression ou un 


\section{DELEUZE ET LES STOÏCIENS}

événement qu'elle ne peut « rationnellement » représenter: l'événement du tout dans lequel la représentation, elle aussi, est déterminée. Deleuze dit très clairement: il y a une différence de nature entre la représentation et l'expression de l'événement qui culmine avec l'opposition de l'objet $=\mathrm{x}$ comme instance identitaire de la représentation, et de la chose $=\mathrm{x}$ comme élément non identifiable de l'expression dans le paradoxe (LS, 170). Autrement dit, il s'agit une fois de plus des paradoxes de l'ensemble de tous les ensembles et du barbier du régiment, ou encore, l'impossibilité de déterminer finalement un ordre cosmique sans supposer quelque chose qui exprime son propre sens, ce qui est le non-sens. En effet, la question qui s'ensuit maintenant pour Deleuze dans son analyse des Stoïciens n'est pas comment ils déterminent l'événement à strictement parler, mais plutôt, comment serait-il possible de vivre cet événement qui ne peut en toute rigueur de termes être qu'interminablement déterminé? Et pour Deleuze, c'est précisément la question à laquelle répond d'une certaine manière l'éthique stoïcienne, en ce qu'elle implique un certain usage des représentations face à l'événement qui arrive effectivement, non pas de Dieu, mais du " dehors ». L'épistémologie des Stoïciens, au lieu de fonder au sens strict le va-et-vient entre la divination physique et son interprétation logique, diffère en effet le problème de l'événement et nous renvoie à leur éthique.

\section{L'éthique}

Dans Logique du sens, Deleuze ne s'intéresse pas explicitement à l'éthique stoïcienne en tant que telle, c'est-à-dire à ses objets particuliers d'étude: les impulsions, les fonctions propres, et cætera. Il s'intéresse plutôt à l'impératif moral chez les Stoïciens, et aux conséquences qui s'ensuivent. Diogène Laërce formule cet impératif de la manière suivante: " vivre en accord avec la nature ", ou « vivre en accord avec l'expérience de ce qui arrive par nature,...car nos natures sont des parties de celle du tout $\gg .^{74}$ Autrement dit, vivre est une activité appartenant à un corps, mais aussi une activité qui doit se conformer à la nature, laquelle, elle aussi, agit et constitue même l'unique agent véritable. ${ }^{75} \mathrm{Et}$ pour que quelqu'un puisse se conformer à la nature, puisse « vouloir »l'événement, il doit évidemment la comprendre. Pour les Stoïciens alors, en ce qui concerne leur éthique, d'une part, il s'agit de l'expérience de vivre ce qui arrive dans son corps et pour son corps, un corps qui agit et pâtit et est cause parfaite des événements qui en dépendent. D'autre part, il s'agit, selon Épictète, d'un certain usage de 


\section{SEAN BOWDEN}

nos représentations, c'est-à-dire, d'interpréter cette expérience par rapport au tout, le kosmos ou la nature. ${ }^{76}$

Or, comme nous venons de le voir, si la nature ne peut jamais arriver toute entière, si la représentation rationnelle, pour être compréhensive et donc digne d'assentiment, doit envelopper un événement qu'elle ne peut représenter, l'impératif moral "vivre en accord avec l'expérience de ce qui arrive par nature » se transforme en " vivre en accord avec l'expérience de l'événement qui n'arrive jamais ", ou plutôt «l'événement avec lequel on n'en finit jamais ». Dans la formulation de Deleuze alors, la « morale stoïcienne. .. consiste à vouloir l'événement comme tel, c'est-à-dire à vouloir ce qui arrive en tant que cela arrive » (LS, 168; c'est moi qui accentue). Ou encore, elle consiste à vouloir " non pas exactement ce qui arrive, mais quelque chose dans ce qui arrive...: l'Événement...le pur exprimé » (LS, 175).

Victor Goldschmidt, que Deleuze suit ici, rétablit le raisonnement derrière cette conception de l'éthique qui est surtout un ars vitae face à l'arrivée de l'événement pur. Premièrement, il faut rappeler que pour les Stoïciens, seul Dieu perçoit la totalité, l'entrelacement total des événements. Mais pour nous, à partir d'un événement donné, nous ne pouvons parvenir à rétablir toutes les liaisons par où il prend sa signification dans l'ensemble. ${ }^{77}$ Et pourtant, deuxièmement, nous sommes bien des "parcelles détachées » de Zeus. ${ }^{78}$ Chaque agent, comme nous l'avons vu, est cause parfaite de tout événement qui dépend de lui, sinon le roulement du cylindre, du moins la poussée qu'il en donne. Comme l'écrit Goldschmidt donc, selon Sénèque, l'agent particulier corporalise pour ainsi dire, ce que nous voudrions appeler vit, les effets incorporels dont on peut dire qu'il est cause. La promenade, par exemple, incorporelle en tant que manière d'être, prend corps sous l'effet du principe hégémonique, ou de la faculté directrice de l'âme, qui s'y manifeste $(\mathrm{LS}, 172) .{ }^{79}$ Mais que veut dire exactement corporaliser ou vivre ainsi l'événement?

D'une part, il s'agit des événements que le corps de l'agent, sous la direction de la partie hégémonique de l'âme, est capable de susciter comme leur cause parfaite (se promener, s'asseoir, et cætera). D'autre part, il s'agit des représentations auxquelles l'âme consent, dans la mesure où il est nécessaire, étant donné notre mode de penser sous la forme " si ceci, alors cela », d'interpréter les liens entre ces événements-ci dont l'agent est cause et le reste du destin. ${ }^{80}$ L'exemple préféré de Deleuze, pour illustrer cette "double causalité » qu'il retire des Stoïciens, est l'événement de mourir, que l'agent doit vivre non seulement dans la 


\section{DELEUZE ET LES STOÏCIENS}

mesure où cet événement s'effectue dans l'intimité de son corps, mais encore en ce sens que cet événement est exprimé dans des propositions qui présentent autant de cas pour comprendre sa signification, c'est-àdire pour suivre la combinaison d'un tel événement avec d'autres événements au niveau du destin. Or, encore une fois, c'est le destin tout entier que l'on ne connaît jamais. Un événement comme « mourir » a donc une structure problématique toujours ouverte (comment, où, quand?), c'est-à-dire dans la mesure où la raison de cet événement, bien qu'il appartienne à l'enchaînement qu'est le destin, est toujours encore à exprimer (LS, 171; 177-78). Comme l'affirme donc Deleuze, inspiré ici par les Stoïciens, un événement comme mourir est toujours déjà passé et encore à venir: fatal mais non-nécessaire. Ou encore, plus précisément:

Dans tout événement, il y a bien le moment présent de l'effectuation, celui où l'événement s'incarne dans un état de choses, un individu, une personne, celui qu'on désigne en disant voilà, le moment est venu; et le futur et le passé de l'événement ne se jugent qu'en fonction de ce présent définitif, du point de vue de celui qui l'incarne. Mais il y a d'autre part le futur et le passé de l'événement pris en luimême, qui esquive tout présent, parce qu'il est libre des limitations d'un état de choses ... sans rapport avec moi, ni avec un moment déterminable comme présent, sauf avec l'instant impersonnel qui se dédouble en encore-futur et déjà-passé...Il y a donc deux accomplissements [de l'événement], qui sont comme l'effectuation et la contreeffectuation. (LS, 177-178)

En «mourant» donc, en concentrant en soi-même la double causalité ouverte de l'événement incorporel, l'agent n'est pas capable, non moins que toute autre " chose ", d'être une substance unificatrice pour ce qui se passe. Il est plutôt un " thème complexe », constitué par les composantes de l'événement auquel il donne son corps, mais où ces composantes sont comprises elles-mêmes comme des événements, libérées de toute instance identitaire capable de les unifier (LS, 176). Mais cela ne veut pas dire que l'agent a effectivement disparu. C'est plutôt précisément en tant quil meurt, par exemple, qu'il « est » quelque chose, de même que tout objet "est » quelque chose en tant que les événements qui lui sont " propres » arrivent, c'est-à-dire en tant que leurs événements sont déterminés de proche en proche dans la structure toujours ouverte qu'est le kosmos, ou plutôt le « chaosmos » comme dit 


\section{SEAN BOWDEN}

Deleuze, où chacun doit se trouver (LS, 205-207).

Deleuze écrit que le sage stoïcien, en vivant l'événement, " est libre de deux façons...: une fois parce que son âme peut atteindre à l'intériorité des causes physiques parfaites, une autre fois parce que son esprit peut jouer des rapports très spéciaux qui s'établissent entre les effets dans un élément de pure extériorité » (LS, 198). Cet « élément de pure extériorité ", comme nous l'avons vu, est l'événement pur, ce qui ne peut jamais être représenté. Donc, le sage stoïcien dans sa liberté " comprend l'événement pur dans sa vérité éternelle, indépendamment de son effectuation spatio-temporelle....Mais, aussi et en même temps, du même coup, il veut l'incarnation, l'effectuation de l'événement pur incorporel dans un état de choses et dans son propre corps » (LS, 172). Et en tant que le sage vit ou corporalise ainsi l'événement, il devient ce que Deleuze appelle la "quasi-cause » de l'événement, il « contre-effectue » l'événement, c'est-à-dire qu'il le représente, le sélectionne, et dirige son effectuation ou son interprétation vivante en tant qu'il arrive (LS, 173). Voilà donc enfin non seulement le lien éthique entre la divination physique et son interprétation logique, mais encore la clef de voûte du système deleuzo-stoïcien du « maniement » de l'événement qu'exige la division irréductible ontologique entre les corps et les incorporels.

\section{Conclusion: Le Stoïcisme de Deleuze}

Nous voyons clairement maintenant l'importance des Stoïciens pour Deleuze. Point après point, ils répondent aux paradoxes de l'événement tels que Deleuze les a analysés. Premièrement, la division stoïcienne ontologique entre les corps et les événements incorporels résout le problème de l'affirmation de l'événement comme objectivement nécessaire pour penser le monde, bien qu'en même temps il ne puisse jamais avoir le statut d'un objet mondial car il implique en lui-même deux états de choses différents. Autrement dit, étant donnée cette distinction ontologique, l'événement peut désormais objectivement subsister dans les propositions qui viennent l'exprimer sans se confondre ni avec la proposition désignatrice ni avec le désigné, ces derniers étant tous les deux des corps. L'événement est ici à la fois comme une idéalité (objective mais problématique) et aussi le sens à venir de ce qui se dit. Deuxièmement, la philosophie stoïcienne répond au paradoxe selon lequel l'événement doit être dit à la fois " cause » et « effet » des états de choses. C'est parce que, d'une part, la différence ontologique entre les corps et les événements incorporels met toute 


\section{DELEUZE ET LES STOÏCIENS}

causalité réelle dans les corps, ce qui veut dire que les événements incorporels ne peuvent « être » que des effets de ces derniers. D'autre part pourtant, l'éthique stoïcienne, lien entre la divination physique et son interprétation logique, pose l'agent rationnel, lui-même un « thème événementiel complexe », comme " quasi-cause » de ce qui se passe. $\AA$ l'intérieur du relais de rapports ouverts mais tigoureux qu'établissent donc les Stoïciens, cette "double causalité » laisse se poser ontologiquement l'événement comme (quasi-)cause et comme effet des états de choses, toujours supposé mais dont la détermination «événementielle » est infiniment différée (ce qui était notre troisième et dernier paradoxe).

Toutefois, pour Deleuze, ayant suivi les Stoïciens aussi loin que possible, et bien quil ne nous en offre pas la preuve, il semble que ceux-ci "n'aient pu conjurer la double tentation de revenir à la simple causalité physique ou àla contradiction logique »(LS, 200). Cela veut dire sans doute, en d'autres termes, que les Stoïciens affirment en dernière analyse un tout, disons un « nom » littéral, Dieu ou le kosmos, en rapport avec lequel aurait pu être déterminée la totalité des événements les uns par rapport aux autres. Comme nous l'avons montré, ce « tout » serait le non-sens etcontraire à ce qu'exige l'événement pur. Ceci a au moins pour résultat, quant à la détermination de la philosophie événementielle de Deleuze, la nécessité de pousser plus loin que sa lecture des Stoïciens. Et pourtant, selon un abrégé récent des concepts deleuziens, il parait que Deleuze conserve toujours certains des éléments de la philosophie stoïcienne tels que nous les avons examinés ci-dessus, même sil cherche pour eux un autre fondement, un fondement qui serait lui-même l'événement pur selon les exigences de la problématique de Logique du sens. toujours un événement derrière un événement. Résumons-les brièvement. Tout d'abord, il garde la différence de nature entre l'événement et tout état de choses fixe. Il conserve également la conception d'un rapport fatal mais non-nécessaire entre événements, c'est-à-dire une espèce de raison suffisante ouverte pour ce qui se passe. C'est l'Un tristement célèbre de Deleuze. Et enfin, il retient lidée d'une responsabilité devant l'événement, ou encore une éthique de la contreeffectuation de l'événement. On peut trouver ces éléments dans des textes aussi divers que Mille Plateaux, Qu'est-ce que la philosopbie?, et Le Pli: Nous ne pouvons évidemment pas suivre ces développements ici. Il suffira peutêtre-disons pour linstant--de les avoir indiqués.

University of New South Wales

Université de Paris VIII, Vincennes—Saint-Denis 


\section{SEAN BOWDEN}

\section{Notes}

Je tiens à exprimer ma gratitude à $\mathrm{M}$. Paul Patton et à M. Todd May pour leurs commentaires sur une version précédente de cet essai. Je suis aussi extrêmement reconnaissant à $\mathbf{M}$. Florentin Roche pour ses conseils linguistiques.

${ }^{1}$ Gilles Deleuze, Logique du sens (Paris: Éditions de Minuit, 1969). Désormais « $\mathrm{LS}$ » dans le texte.

${ }^{2}$ Selon la formule dans Gilles Deleuze, Différence et répétition (Paris: PUF, 1968), 304305.

${ }^{3}$ Deleuze, Différence et répétition, 304-305.

${ }^{4}$ Nous voyons ici en effet la signification de la critique deleuzienne du Platonisme à travers une lecture du Sophiste, c'est-à-dire où la « définition finale du sophiste nous mène au point où nous ne pouvons plus le distinguer de Socrate lui-même " (LS, 295). Autrement dit, cette définition montre que chaque tentative de définir un état de choses, soit le Sophiste, soit le vrai prétendant ou la bonne copie, soit l'authentification mythique de l'Idée à la manière du Phèdre ou du Politique, suppose un événement l'instant logiquement insurmontable ou infiniment différé de son établissement. Une fois de plus, dire ce qu'est quelque chose implique au moins aussi ce qu'est « dire ».

${ }^{5}$ Michel Foucault, "Theatrum Philosophicum, " Critique, no. 282 (novembre 1970): 885-908.

${ }^{6}$ A.A. Long et D.N. Sedley, Lesphilosophes hellénistiques - II Les Stoïciens, trad. Jacques Brunschwig et Pierre Pellegrin, (Paris: Flamarion, 2001), \$27, §51B (Désormais « PH»). Sextus Empiricus, Contre lesprofesseurs X, 218 (extrait partiel de SVFII, 331). Alexandre, Sur les'Topiques d'Aristote 301, 19-25 (SVF II, 329). Galien, De la méthode médicale X, 155, 1-8 (extrait partiel de SVF II, 322). Stobée I, 106, 5-23 (SVF II, 510).

${ }^{7} \mathrm{PH}, \llbracket 30 \mathrm{~A}$, \27C. Stobée I, 136, 21-137, 6 (SVF I, 65). Sextus Empiricus, Contre les professeurs I, 17 (SVF II, 330).

${ }^{8} \mathrm{PH}$, \44B et E-F, §45A. Diogène Laërce VII, 134 (extrait partiel de SVFII, 300, plus II, 299); VII, 137 (extrait partiel de SVFII, 526). Calcidius 293 (extrait partiel de SVF I, 88). Cicéron, Académiques I, 39 (SVF I, 90).

${ }^{9} \mathrm{PH}, \$ 45 \mathrm{C}$. Némésius 78, 7-79, 2 (extrait partiel de $S V F \mathrm{I}, 518$ ).

${ }^{10} \mathrm{PH}, \$ 60$; 33E; 28M; 61B. Sénèque, Lettres 117, 2; 13. Simplicius, Sur les Catégories d'Aristote 214, 24-37 (extrait partiel de SVFII, 391). Plutarque, De la vertu morale 440E-441D.

${ }^{11} \mathrm{PH}$, 『47J, \$48C. Némésius 70, 6-71, 4. Alexandre d'Aphrodise, Du mélange 216, 14-218, 6 (SVF II 473).

${ }^{12} \mathrm{PH}$, \55E, G, N. Sénèque, Lettres 65, 2. Aétius I, 11, 5 (SVF II, 340). Alexandre d'Aphrodise, Du destin 191, 30-192, 28 (SVF II, 945).

${ }^{13} \mathrm{PH}$, \27D, \45B. Sextus Empiricus, Contre les professeurs X, 218 (extrait partiel de SVF II, 331); VIII, 263 (SVF II, 363). 


\section{DELEUZE ET LES S'TOÏCIENS}

${ }^{14}$ Victor Goldschmidt, Le système stö̈cien et lidée du temps (Paris: Vrin, 1953), 28. PH, \$49C, B. Cléomède 8, 10-14 (SVF II, 541). Sextus Empiricus, Contre les professeurs X, 3-4 (extrait partiel de SVFII 505). SVF II 505).

${ }^{15} \mathrm{PH}$, , $49 \mathrm{~B}$. Sextus Empiricus, Contreles professeurs X, 3-4 (extrait partiel de

${ }^{16}$ Jean Brun, Le stoïcisme (Paris: PUF, 1958), 56.

${ }_{17}$ Arius Didyme, 26 (SVF II, 509), dans Goldschmidt, Le système stoïcien, 30-31. PH §51A, B. Simplicius, Sur les Catégories d'Aristote, 350, 15-16 (extrait partiel de SVF II, 510). Stobée I, 106, 5-23 (SVF II, 509).

${ }^{18}$ Voir Goldschmidt, Le système stoïcien, 30-48. 341).

${ }^{19} \mathrm{PH}$, \55B. Sextus Empiricus, Contre les professeurs IX, 211 (SVFII,

${ }^{20} \mathrm{PH}, \$ 55$ (commentaire).

${ }^{21}$ Goldschmidt, Le système stoïcien, 30.

${ }^{22} \mathrm{PH}$, \$26B. Diogène Laërce VII, 39-41.

${ }^{23} \mathrm{PH}, \$ 60 \mathrm{~A}, \$ 31 \mathrm{C}, \$ 31 \mathrm{~B}, \$ 64 \mathrm{H}$. Plutarque, Des contradictions des Stö̈ciens, 1035C-D (SVFIII, 68). Diogène Laërce VII, 83 (SVF II, 130); VII, 46-48 (extrait partiel de SVFII, 130). Cicéron, Des termes extrêmes des biens et des maux III, 24-25 (SVF III, 111, extrait partiel).

${ }^{24} \mathrm{PH}$, \$26A. Aétius I, Préface 2 (SVF II, 35). 1989), 54.

${ }^{25}$ Jean-Joël Duhot, La conception stoöcienne de la causalité (Paris: Vrin,

${ }^{26}$ Duhot, La conception stoïcienne de la causalité, 61.

${ }^{27}$ Émile Bréhier, La théorie des incorporels dans l'ancien stö̈icsme (Paris: Vrin, 1928), 11-13. Voir aussi, Goldschmidt, Le système stoïcien, 15-16.

${ }^{28}$ Voir, Duhot, La conception stöicienne de la causalité, 65 . Simplicius, Sur la Physique d'Aristote. II, 2, Diels, pp.291 sq.

${ }^{29} \mathrm{PH}, \S 55 \mathrm{~N}$. Alexandre d'Aphrodise, Du destin 191, 30-192, 28 (SVF II, 945).

${ }^{30}$ Michael Frede, "The Original Notion of a Cause," dans Essays in Ancient Pbilosopby (Minneapolis: University of Minnesota Press, 1987), 128.

${ }^{31}$ Bréhier, La théorie des incorporels, p.6.

${ }^{32} \mathrm{PH}$, \55A. Stobée I, 138, 14139, 4 (SVF I, 89 et II, 336).

${ }^{33} \mathrm{PH}$, §55C. Clément d'Alexandrie, Mélanges VIII, 9, 26, 3-4.

${ }^{34} \mathrm{PH}$, \$55B. Sextus Empiricus, Contre les professeurs IX, 211 (SVFII, 341). Frede, "The Original Notion of a Cause," 137.

${ }^{35} \mathrm{PH}$, §55N. Alexandre d'Aphrodise, Du destin, 191, 30-192, 28 (SVF II, 945).

${ }^{36}$ Frede, "Original Notion of Cause," 149.

${ }^{37} \mathrm{PH}$, \$62C, D. Cicéron, Du destin 39-43 (SVF II, 974). Aulu-Gelle VII, 2, 6-13 (SVF II, 1000, extrait partiel).

${ }^{38}$ Comme montre la critique de Chrysippe de "l'Argument Paresseux ». PH, §55S. Cicéron, $D u$ destin 28-30. 


\section{SEAN BOWDEN}

${ }^{39} \mathrm{PH}$, \33E. Sénèque, Lettres $117,13$.

${ }^{40} \mathrm{PH}$, §33A, C. Diogène Laërce VII, 57 (extrait partiel de SVFIII, Diogène 20). Sextus Empiricus, Contre les professeurs VIII, 70 (extrait partiel de SVF II, 187).

${ }^{41} \mathrm{PH}$, @33B. Sextus Empiricus, Contre les professeurs VIII, 11-12 (extrait partiel de $S V F$ II, 166).

${ }^{42} \mathrm{PH}$, 『33F-G. Diogène Laërce VII, 63-64.

${ }^{43} \mathrm{PH}$, §34A. Diogène Laërce VII, 65.

${ }^{44} \mathrm{PH}$, 〔35A. Diogène Laërce VII, 71-74.

${ }^{45} \mathrm{PH}$, §35D. Aulu-Gelle XVI, 8, 10-11 (extrait partiel de FDS 967). Voir aussi: Jacques Brunschwig, "Le modèle conjonctif, » dans Études sur les philosopbies hellénistiques - Épicurisme, stö̈isme, scepticisme (Paris: PUF, 1995), 161187.

${ }^{46} \mathrm{PH}$, \35E, Aulu-Gelle XVI, 8, 12-14 (FDS 876).

${ }^{47} \mathrm{PH}$, \35A. Diogène Laërce VII, 71-74.

${ }^{48}$ Voir pour les références à cette discussion PH, p.115, n.1.

${ }^{49} \mathrm{PH}$, [53T. Sextus Empiricus, Contre les professeurs VIII, 275-276 (extrait partiel de SVF II, 223).

50 Deleuze s'inspire peut-être ici d'autres commentateurs sur les Stoïciens. Voir, par exemple, Pierre-Maxime Schuhl, Le dominateur et les possibles (Paris: PUF, 1960), 85-86.

${ }^{51}$ Deleuze refuse évidemment ici la solution axiomatique aux paradoxes de la théorie des ensembles.

${ }^{52} \mathrm{PH}$, \38E. Cicéron, Du destin 12-15.

${ }^{53}$ Voir PH, §34E-F. Diogène Laërce VII, 65 (extrait partiel de SVF II, 193). Sextus Empiricus, Contre les professeurs VIII, 103. Voir, pourtant, Brunschwig, qui n'est pas sûr quel'on peut interpréter ainsi les transformations des conditionnels chaldéens: « Le modèle conjonctif », 187, n.2.

${ }^{54} \mathrm{PH}$, [53'T. Sextus Empiricus, Contre les professeurs VIII, 275-276 (extrait partiel de $S V F$ II, 223).

${ }^{55} \mathrm{PH}$, \51H. Sextus Empiricus, Contre les professeurs VIII, 254-255 (extrait partiel de SVF II, 221). Goldschmidt, Le système stoïien, 43-44.

${ }^{56}$ Voir Goldschmidt, Le système stoïcien, 81-83.

${ }^{57}$ Voir $\mathrm{PH}$, \55S. Cicéron, Du destin 28-30.

${ }^{58} \mathrm{PH}$, @34D. Sextus Empiricus, Contre les professeurs VIII, 85-86.

${ }^{59} \mathrm{PH}$, §31C; 53T. Diogène Laërce VII, 83 (SVF II, 130). Sextus Empiricus, Contre les professeurs VIII, 275-276 (extrait partiel de SVF II, 223).

${ }^{60} \mathrm{Je}$ vais conserver la traduction proposée par Goldschmidt de phantasia en "représentation", par souci de correspondance avec le vocabulaire employé par Deleuze. Il est cependant important de rappeler que les phantasiai sont toutes corporelles.

${ }^{61} \mathrm{PH}$, [39B. Aétius IV, 12, 1-5 (extrait partiel de SVFII, 54).

${ }^{62}$ Voir Michael Frede, "Stoic Epistemology, » dans The Cambridge 


\section{DELEUZE ET LES STOÏCIENS}

History of Hellenistic Pbilosopby, sous la direction de Keimpe Algre et autres (Cambridge: Cambridge University Press, 1999), 304-305.

${ }^{63} \mathrm{PH}$, 333C-D. Sextus Empiricus, Contre les professeurs VIII, 70 (extrait partiel de SVFII, 187). Diogène Laërce VII, 49 (extrait partiel de SVFII, 52).

${ }^{64} \mathrm{PH}$, \39D; 53T; 63E. Diogène Laërce VII, 53 (extrait partiel de SVFII, 87). Sextus Empiricus, Contre les professeurs VIII, 275-276 (extrait partiel de SVF II, 223). Épictète, Entretiens I, 6, 12-22.

${ }^{65}$ Frede, «Stoic Epistemology, » 306-307. PH, \39A; C. Diogène Laërce VII, 49-51 (SVF II 52, 55, 61). Cicéron, Académiques II, 21.

${ }^{66} \mathrm{PH}$, §39E. Aétius IV, 11, 1-4 (SVF II, 83).

${ }^{67}$ Frédérique Ildefonce, Les stoïciens I - Zénon, Cléanthe, Chrysippe (Paris: Les Belles Lettres, 2000), 102. Ce texte très clair n'est pas sans rapport avec la lecture que je présente ici du stoïcisme de Deleuze. Voir la discussion du stoïcisme " sans cosmos » de Nietzsche, 204209. Voir aussi, sur le rapport entre les représentations sensibles et la dialectique stoïcienne, Claude Imbert, "Un platonisme bien tempéré: la dialectique stoïcienne, " dans Pour une bistoire de la logique - un béritage platonicien (Paris, PUF, 1999), 89-123.

${ }^{68} \mathrm{PH}$, \40A. Diogène Laërce VII, 54 (incluant SVF II, 105 et le fr. 42 de Posidonius).

${ }^{69} \mathrm{PH}$, \40E. Sextus Empiricus, Contre les professeurs VII, 247-252 (extrait partiel de SVF II, 65).

${ }^{70}$ Frede, «Stoic Epistemology, » 302-303. $\mathrm{PH}$, §34D.

${ }^{71}$ Question de Sextus Empiricus, Contre les professeurs VIII, 85-86.

${ }^{72}$ Sur la thèse stoïcienne de l'identité des indiscernables, voir $\mathrm{PH}$, §40J. Cicéron, Académiques II, 83-85.

${ }^{73}$ Frede, "Stoic Epistemology, » 308-310; 314.

${ }^{74} \mathrm{PH}$, §63C. Diogène Laërce VII, 87-89.

${ }^{75}$ Goldschmidt, Le système stoïcien, 77.

${ }^{76} \mathrm{PH}$, §62K; 63E. Épictète, Entretiens I, 1, 7-12; I, 6, 12-22.

${ }^{77}$ Goldschmidt, Le système stoïcien, 97-98.

${ }^{78}$ Marc Aurèle, V, 27.

${ }^{79} \mathrm{PH}$, [53L. Sénèque, Lettres 113,23 . Goldschmidt, Le système stoïcien, 107.

${ }^{80}$ Goldschmidt, Le système stoïcien, 107-108. PH, \$62K; 63E. Épictète, Entretiens I, 1, 7-12; I, 6, 12-22.

${ }^{81}$ Robert Sasso, "Événement (Pur), " dans Le vocabulaire de Gilles Deleuze, sous la direction de Robert Sasso et Arnaud Villani (Paris: Vrin, 2003), 138-153. Voir aussi François Zourabichvili, «Événement, » dans Le vocabulaire de Deleure (Paris: Ellipses, 2003), 36-40. 